\title{
Accurate Monitoring of Parkinson's Disease Symptoms With a Wearable Device During COVID-19 Pandemic
}

\author{
KONSTANTINOS I. TSAMIS ${ }^{1}$, GEORGE RIGAS ${ }^{2,3}$, KOSTIKIS NIKOLAOS ${ }^{3}$, \\ DIMITRIOS I. FOTIADIS ${ }^{2}$ and SPYRIDON KONITSIOTIS ${ }^{1}$ \\ ${ }^{1}$ Department of Neurology, University Hospital of Ioannina, Ioannina, Greece; \\ ${ }^{2}$ Unit of Medical Technology and Intelligent Information System, \\ Department of Materials Science and Engineering, University of Ioannina, Ioannina, Greece; \\ ${ }^{3}$ PD Neurotechnology Ltd, Ioannina, Greece
}

\begin{abstract}
Background: Accurate assessment of symptoms in Parkinson's disease $(P D)$ is essential for optimal treatment decisions. During the past few years, different monitoring modalities have started to be used in the everyday clinical practice, mainly for the evaluation of motor symptoms. However, monitoring technologies for PD have not yet gained wide acceptance among physicians, patients, and caregivers. The COVID-19 pandemic disrupted the patients' access to healthcare, bringing to the forefront the need for wearable sensors, which provide effective remote symptoms' evaluation and follow-up. Case Report: We report two cases with PD, whose symptoms were monitored with a new wearable $C E$ marked system (PDMonitor $\left.{ }^{\circledR}\right)$, enabling appropriate treatment modifications. Conclusion: Objective assessment of the patient's motor symptoms in his daily home environment is essential for an accurate monitoring in PD and enhances treatment decisions.
\end{abstract}

During the last years, wearable sensors and remote monitoring systems have been used in healthcare for monitoring symptoms and activity of patients in their everyday environment and this technology is constantly improving to meet patients' needs and physicians' expectations $(1,2)$. In advanced Parkinson's disease (PD), parkinsonian symptoms gradually exacerbate and the response to levodopa changes, contributing to the emergence

This article is freely accessible online.

Correspondence to: Konstantinos I. Tsamis, Department of Neurology, University Hospital of Ioannina, GR 45110 Ioannina, Greece. Tel: +30 2651007577, e-mail: ktsamis@uoi.gr

Key Words: Parkinson's disease, remote monitoring, wearables, fluctuations, objective measurements. of motor fluctuations. Motor fluctuations are characterized by the transition between periods of good symptom control and others with poor symptom control. Accurate assessment of these fluctuations is crucial for the optimal treatment decisions that can alleviate the problem (3-5).

However, the current diagnostic approach poses difficulties in a thorough evaluation of the duration of periods with satisfactory motor performance and others with prominent parkinsonian symptoms, due to inadequate information acquired from in-person (or virtual) patient encounters and low reliability of self-reports and diaries (6). In response to this issue, several monitoring systems have been developed so far to provide the means for telehealth in PD and other neurodegenerative diseases (7). Wearable devices can offer prolonged objective measurements of motor symptoms (8). Sensors that are currently in use, can help in the evaluation of motor symptoms in patients' home environment and have shown promising results in the care of patients with PD $(9,10)$. Nonetheless, disadvantages of these technologies still exist, like the fact that each device has shown satisfactory results only in the detection of specific symptoms and not sufficient correlation with patients' diaries $(11,12)$, thus further enhancements and clinical validation will guarantee the applicability of wearable technologies in everyday clinical practice $(13,14)$. Especially, in the era of the COVID-19 pandemic that disrupted the patients' access to healthcare, poor follow-up of the patients aggravates the difficulties on the clinical evaluation and threatens to deteriorate their quality of life $(15,16)$. The present report includes two cases with advanced PD and troublesome fluctuations whose motor symptoms were monitored with PDMonitor ${ }^{\circledR}$, a wearable monitor system, accompanied by a mobile app for medication adherence and patient reported events, that can detect and monitor all motor manifestations of PD and the patients' daily activity. 


\section{Case Presentation}

Case 1. A 70-year-old male patient diagnosed with PD eight years ago and on levodopa treatment for the last seven years, presented reporting exacerbation of the symptoms during the afternoon and excess fatigue, making it difficult for him to continue his activities. During the last years the patient was stable, and was even able to continue working, receiving high doses of levodopa (1,000 mg per day), an inhibitor of monoamine oxidase- $\mathrm{B}$ rasagiline (1 mg per day), and dopamine agonists rotigotine ( $8 \mathrm{mg}$ per day) and ropinirole ( $8 \mathrm{mg}$ per day). Initial assessment of the patient in the outpatient department revealed only mild parkinsonian symptoms, and despite intensive effort to acquire an accurate history, self-reported information about symptoms was inadequate to lead into a definite diagnosis. Since hospitalization could not be addressed for symptoms monitoring, due to restrictions applied during the COVID19 pandemic, the use of PDMonitor ${ }^{\circledR}$ was proposed, for assessment of the symptoms in the patient's daily environment throughout the daytime. The patient wore the system consisting of wearable sensors for two days and in parallel, used the mobile application to demarcate the time periods of symptoms' worsening. The report produced by the device (Figure 1) revealed that the patient experienced rapid transitions between "ON" and "OFF" periods, with exacerbation of bradykinesia, freezing of gait, and gait impairment during the "OFF" time. These motor complications were more pronounced during the afternoon and appeared to have perfect match with the periods of symptoms' worsening marked by the patient. With the aid of the PDMonitor ${ }^{\circledR}$ report, the doses of levodopa were increased from five per day to six per day, and apomorphine injections were introduced to cover the intermediate periods with sudden and unpredictable changes in the symptoms. Finally, the medication's changes effectively addressed patient's symptoms and improved his quality of life.

Case 2. The second case is a female patient, 73 years old, diagnosed with PD four years ago and on levodopa treatment from her initial diagnosis. The patient had also a long history of depression, treated during periods of symptoms' exacerbations with serotoninergic medications. The last months, after a stressful event, the patient experienced sudden recurrent episodes of intense fear and anxiety accompanied by shortness of breath and restlessness. The initial diagnosis was panic disorder and different medications (serotonin reuptake inhibitors, benzodiazepines and neuroleptics) were applied to address it, without any significant improvement. Clinical evaluation of the patient during one of the episodes revealed significant deterioration of movements with increased bradykinesia and rigidity, along with anxiety and fear. Thus, a close monitoring was proposed for the patient to assess the nature of the symptoms. Social distancing and restrictions in the access to healthcare units, due to the COVID-19 pandemic, posed difficulties in an in-person long lasting evaluation. The use of PDMonitor ${ }^{\circledR}$ was chosen instead, for continuous monitoring of motor symptoms during daytime. The report created by the system (Figure 2) was highly correlated with exacerbation events as those reported by the caregiver. The evaluation showed a perfect match of the panic attacks with periods of re-emergence of parkinsonian motor symptoms and the diagnosis of non-motor wearing off was set, with prominent changes in mood, anxiety, fear, and restlessness. The gradual increase of daily levodopa dose from $300 \mathrm{mg}$ to $900 \mathrm{mg}$, along with the initial treatment of benzodiazepines and neuroleptics, led to the reduction in the frequency of the episodes and alleviation of the symptoms.

\section{Discussion and Conclusion}

The treatment for PD is currently focused on the optimal management of motor and non-motor symptoms, since there is no therapy up to date, with proven efficacy on neuroprotection or modification of disease progression (4). For physicians, accurate information about the symptomatology of the patients throughout the day are indispensable, in order to reach the right treatment decisions (5). Technology-empowered precision medicine is offering nowadays the means for accurate monitoring of the patients and wearable devices are used frequently in the everyday clinical practice (17). During the COVID-19 pandemic, these technologies seem to gain more ground, offering a reliable monitoring remotely, and abridging the time needed for inperson clinical examination (18).

Assessment of symptoms in advanced PD is very often a challenging task, due to the diversity of motor and nonmotor symptoms and the rapid transitions between "ON" and "OFF" periods. Besides, the time of in-person clinical evaluation is usually limited, giving only a partial view of the total clinical manifestations, and traditional means like self-reported symptoms, diaries and caregivers' descriptions, aim to bridge the gap of missing information. However, these means are very often inadequate and even worse, they may mislead physicians, in case of poor descriptions caused by memory limitations, wrong interpretations of the symptoms' nature, and over- or under-estimation of symptoms due to mood disorders (19).

The two cases presented here are illustrative examples of the potential of a wearable monitoring system to capture accurately the diverse clinical manifestations of advanced $\mathrm{PD}$, reducing the need for prolonged in-person examination or hospitalization. Both cases presented, had significant difficulties in the diagnostic approach posed due to missing information on the time course of symptoms throughout the 


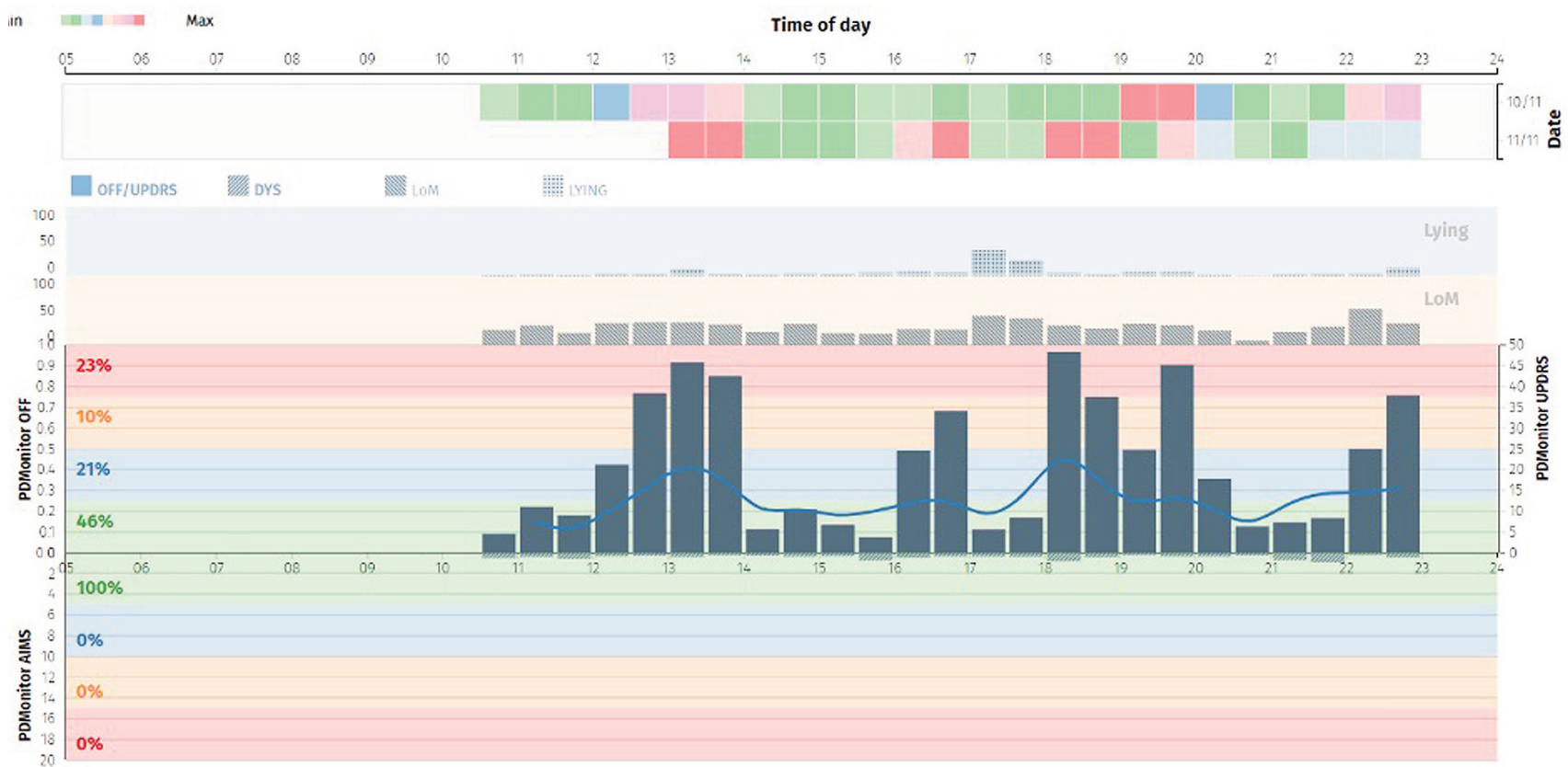

Figure 1. Report diagram from PDMonitor ${ }^{\circledR}$ showing the total 'OFF' score of the patient calculated by the system for a selected period, expressed as a score between 0 and 1. The report shows transitions between an 'ON' and 'OFF' state and exacerbation during afternoon.

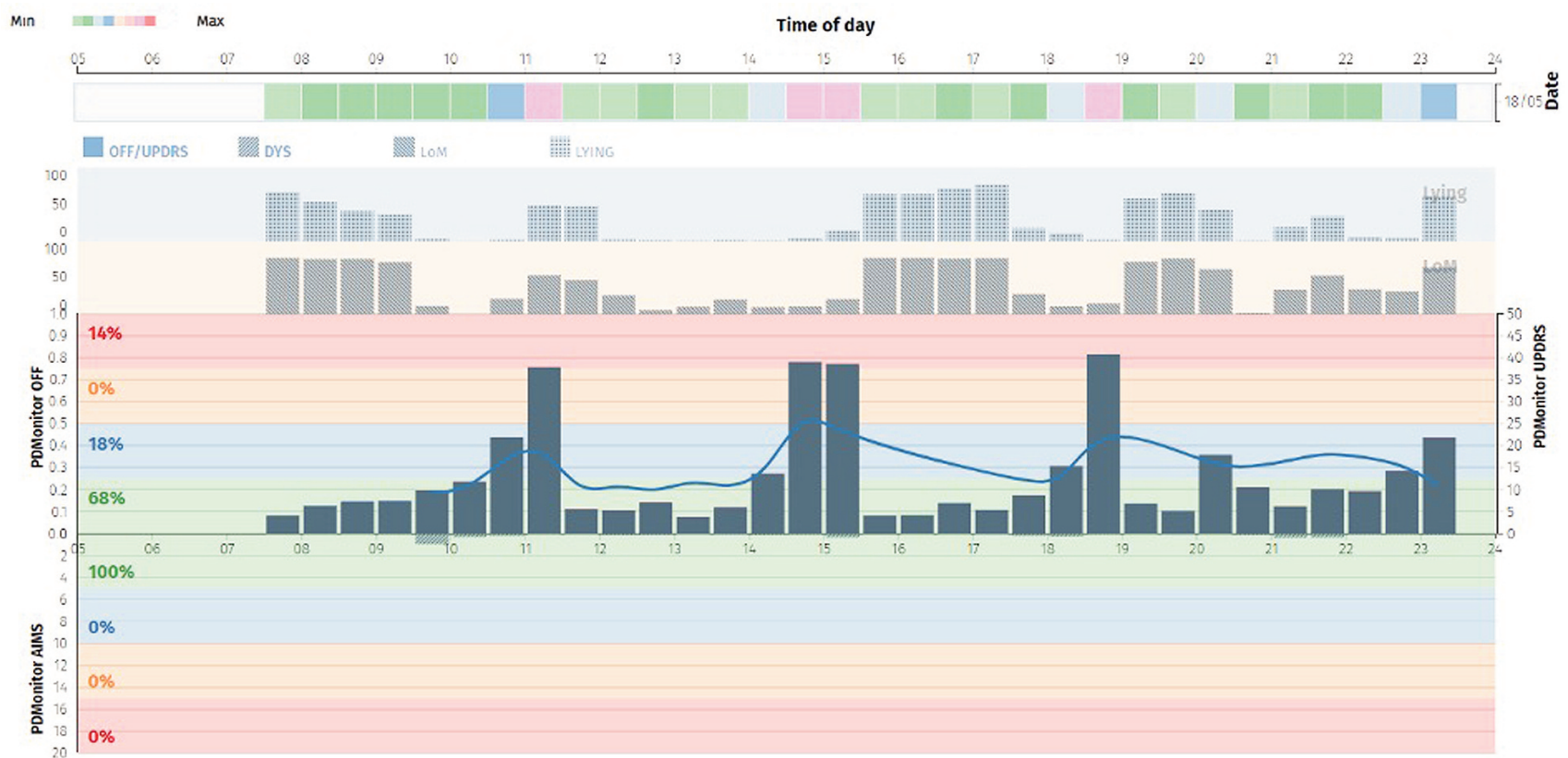

Figure 2. Report diagram from PDMonitor ${ }^{\circledR}$ showing the total 'OFF' score of the patient calculated by the system for a selected period, expressed as a score between 0 and 1. The report shows periods with a high 'OFF' score that were highly correlated with exacerbation events reported by the caregiver. 
day. With the use of PDMonitor ${ }^{\circledR}$, physicians had access to an objective assessment of the patient's motor symptoms as those manifested in his daily home environment and managed to reach a final diagnosis and make the right treatment decisions.

\section{Conflicts of Interest}

D.I.F. and S.K. are the inventors of PDMonitor ${ }^{\circledR}$ and the founders of PD Neurotechnology Ltd. (the manufacturer of the PDMonitor ${ }^{\circledR}$ ). K.I.T., G.R. and N.K. have received salaries from PD Neurotechnology LTD to develop new features for the PDMonitor ${ }^{\circledR}$ and they did not receive any personal or laboratory funding to undertake this study.

\section{Authors' Contributions}

K.I.T. collected the data and wrote the manuscript. G.R. and N.K. interpreted the results from the PDMonitor ${ }^{\circledR}$ reports. N.K. reviewed the bibliography and contributed to the discussion of recent references. D.I.F. and S.K. contributed to the writing and reviewed the manuscript.

\section{References}

1 Huang J, Zhou J, Luo Y, Yan G, Liu Y, Shen Y, Xu Y, Li H, Yan L, Zhang G, Fu Y and Duan H: Wrinkle-enabled highly stretchable strain sensors for wide-range health monitoring with a big data cloud platform. ACS Appl Mater Interfaces 12(38): 43009-43017, 2020. PMID: 32856459. DOI: $10.1021 /$ acsami.0c11705

2 Ye S, Feng S, Huang L and Bian S: Recent progress in wearable biosensors: From healthcare monitoring to sports analytics. Biosensors (Basel) 10(12): 205, 2020. PMID: 33333888. DOI: 10.3390/bios10120205

3 Iijima M, Mitoma H, Uchiyama S and Kitagawa K: Long-term monitoring gait analysis using a wearable device in daily lives of patients with Parkinson's disease: the efficacy of selegiline hydrochloride for gait disturbance. Front Neurol 8: 542, 2017. PMID: 29114238. DOI: 10.3389/fneur.2017.00542

4 Moore H, Shpiner DS and Luca CC: Management of motor features in advanced Parkinson disease. Clin Geriatr Med 36(1): 43-52, 2020. PMID: 31733701. DOI: 10.1016/j.cger.2019.09.010

5 Aradi SD and Hauser RA: Medical management and prevention of motor complications in Parkinson's disease. Neurotherapeutics 17(4): 1339-1365, 2020. PMID: 32761324. DOI: 10.1007/s13311020-00889-4

6 Rastgardani T, Armstrong MJ, Gagliardi AR and Marras C: Understanding, impact, and communication of "off" periods in Parkinson's disease: A scoping review. Mov Disord Clin Pract 5(5): 461-470, 2018. PMID: 30515435. DOI: $10.1002 / \mathrm{mdc} 3.12672$

7 De Marchi F, Contaldi E, Magistrelli L, Cantello R, Comi C and Mazzini L: Telehealth in neurodegenerative diseases: opportunities and challenges for patients and physicians. Brain Sci 11(2): 237, 2021. PMID: 33668641. DOI: 10.3390/brainsci11020237

8 Channa A, Popescu N and Ciobanu V: Wearable solutions for patients with Parkinson's disease and neurocognitive disorder: a systematic review. Sensors (Basel) 20(9): 2713, 2020. PMID: 32397516. DOI: $10.3390 /$ s20092713
9 Bayés À, Samá A, Prats A, Pérez-López C, Crespo-Maraver M, Moreno JM, Alcaine S, Rodriguez-Molinero A, Mestre B, Quispe $\mathrm{P}$, de Barros AC, Castro R, Costa A, Annicchiarico R, Browne P, Counihan T, Lewy H, Vainstein G, Quinlan LR, Sweeney D, ÓLaighin G, Rovira J, Rodrigue Z-Martin D and Cabestany J: A "HOLTER" for Parkinson's disease: Validation of the ability to detect on-off states using the REMPARK system. Gait Posture 59: 1-6, 2018. PMID: 28963889. DOI: 10.1016/j.gaitpost.2017.09.031

10 Joshi R, Bronstein JM, Keener A, Alcazar J, Yang DD, Joshi M and Hermanowicz N: PKG movement recording system use shows promise in routine clinical care of patients with Parkinson's disease. Front Neurol 10: 1027, 2019. PMID: 31632333. DOI: 10.3389/fneur.2019.01027

11 Gao C, Smith S, Lones M, Jamieson S, Alty J, Cosgrove J, Zhang P, Liu J, Chen Y, Du J, Cui S, Zhou H and Chen S: Objective assessment of bradykinesia in Parkinson's disease using evolutionary algorithms: clinical validation. Transl Neurodegener 7: 18, 2018. PMID: 30147869. DOI: 10.1186/s40035-018-0124-x

12 Höglund A, Hagell P, Broman JE, Pålhagen S, Sorjonen K, Fredrikson $\mathrm{S}$ and Svenningsson P: Associations between fluctuations in daytime sleepiness and motor and non-motor symptoms in Parkinson's disease. Mov Disord Clin Pract 8(1): 44-50, 2020. PMID: 33426158. DOI: 10.1002/mdc3.13102

13 Dorsey ER, Bloem BR and Okun MS: A New Day: The role of telemedicine in reshaping care for persons with movement disorders. Mov Disord 35(11): 1897-1902, 2020. PMID: 32870517. DOI: $10.1002 / \mathrm{mds} .28296$

14 Ancona S, Faraci FD, Khatab E, Fiorillo L, Gnarra O, Nef T, Bassetti CLA and Bargiotas P: Wearables in the home-based assessment of abnormal movements in Parkinson's disease: a systematic review of the literature. J Neurol, 2021. PMID: 33409603. DOI: 10.1007/s00415-020-10350-3

15 Prasad S, Holla VV, Neeraja K, Surisetti BK, Kamble N, Yadav $\mathrm{R}$ and Pal PK: Parkinson's disease and COVID-19: perceptions and implications in patients and caregivers. Mov Disord 35(6): 912-914, 2020. PMID: 32304118. DOI: 10.1002/mds.28088

16 Motolese F, Magliozzi A, Puttini F, Rossi M, Capone F, Karlinski K, Stark-Inbar A, Yekutieli Z, Di Lazzaro V and Marano M: Parkinson's disease remote patient monitoring during the COVID-19 lockdown. Front Neurol 11: 567413, 2020. PMID: 33117262. DOI: 10.3389/fneur.2020.567413

17 Gatsios D, Antonini A, Gentile G, Marcante A, Pellicano C, Macchiusi L, Assogna F, Spalletta G, Gage H, Touray M, Timotijevic L, Hodgkins C, Chondrogiorgi M, Rigas G, Fotiadis DI and Konitsiotis S: Feasibility and utility of mHealth for the remote monitoring of Parkinson disease: Ancillary study of the PD_manager randomized controlled trial. JMIR Mhealth Uhealth 8(6): e16414, 2020. PMID: 32442154. DOI: 10.2196/16414

18 Mulroy E, Menozzi E, Lees AJ, Lynch T, Lang AE and Bhatia KP: Telemedicine in movement disorders: Leçons du COVID19. Mov Disord 35(11): 1893-1896, 2020. PMID: 32881108. DOI: $10.1002 / \mathrm{mds} .28297$

19 Armstrong MJ and Okun MS: Diagnosis and treatment of Parkinson disease: a review. JAMA 323(6): 548-560, 2020. PMID: 32044947. DOI: 10.1001/jama.2019.22360

Received April 16, 2021

Revised May 3, 2021

Accepted May 4, 2021 